\title{
Use of components analysis to identify internal heat in breast dynamic thermal images
}

This paper was downloaded from TechRxiv (https://www.techrxiv.org).

\section{LICENSE}

CC BY 4.0

SUBMISSION DATE / POSTED DATE

23-06-2021 / 28-06-2021

\section{CITATION}

Gershenson, Meir (2021): Use of components analysis to identify internal heat in breast dynamic thermal images. TechRxiv. Preprint. https://doi.org/10.36227/techrxiv.14832351.v1

$\mathrm{DOI}$

10.36227/techrxiv.14832351.v1 


\title{
Use of components analysis to identify internal heat in breast dynamic thermal images
}

\author{
Meir Gershenson
}

\begin{abstract}
I suggest a method for biomedical imaging with heat using principal and independent components analysis. This method produces novel results suggesting physiologic mechanisms. When using thermal imaging to detect breast cancer, the dominant heat signature is of indirect heat transported by the blood away from the tumor location into the skin. Interpretation is usually based on vascular patterns and not by observing the direct cancerous heat. In this new method one uses a sequence of thermal images of the patient's breast following external temperature change. Data are recorded and analyzed using independent component analysis (ICA) and principal component analysis (PCA). ICA separates the image sequence into new independent images having a common characteristic time behavior. Using the Brazilian visual lab mastology data set, I observed three types of component images: Images corresponding to a minimum change as a function of applied temperature or time, which suggests an association with the cancer generated heat, images in which a moderate temperature dependence is associated with veins affected by vasomodulation, and images of complex time behavior indicating heat absorption due to high perfusion of the tumor. All components appear to clearly and distinctly represent underlying physiology.
\end{abstract}

Index Terms - Breast cancer, components analysis, dynamic thermogram, vasomodulation, veins.

\section{INTRODUCTION}

Breast cancer is the leading cancer among woman. Early detection is crucial in curing breast cancer. Most common detection methods are mammography, ultrasound, and MRI. Mammography is regarded as the gold standard in breast cancer screening. However, mammography has certain limitations: Mammography is less effective in dense breast, mammography requires $\mathrm{X}$ radiation, which is expected to increase the prevalence of cancer, the test is quite uncomfortable, and the cost is high for developing countries.

Thermography is based on use of medium or long wave infrared (IR) cameras. The body, like most physical objects, emits IR radiation depending on the temperature. Taking an IR image of the body is equivalent to mapping the body temperature. While thermography was approved by the FDA as an adjunct method to mammography in 1982, its utility is very limited due to low sensitivity and low ability to distinguish between healthy and diseased tissue. Cancer is characterized by a high metabolic rate, which causes an increase in the temperature. The high metabolic rate requires an increased blood supply, which is compensated by enlarging existing blood vessels and creating new ones. This process is called angiogenesis. The increase of blood flow at the cancerous site is associated with an increase in the tissue's ability to absorb heat. The bio heat transfer equation describing thermal propagation is given by ${ }^{1}$

$$
\begin{array}{r}
\nabla k(\boldsymbol{r}) \nabla T(\mathbf{r}, t)-\rho C \cdot \frac{\partial}{\partial t} T(\boldsymbol{r}, t)-Q b+Q m=0 \\
Q b=\omega C b\left[T(\mathbf{r}, t)-T_{0}(\mathbf{r}, t)\right]
\end{array}
$$

Typical values are taken from Azarnoosh $\mathbf{J}$ et al. ${ }^{2}$ and are given in Table 1. From the above table the increase in blood

TABLE I

THRMOPHYSICAL PARAMETERS OF TYPICAL BREAST

\begin{tabular}{|c|c|c|c|c|c|}
\hline \hline & $\begin{array}{c}\text { Density } \\
\rho \\
(\mathrm{kg} / \mathrm{m} 3)\end{array}$ & $\begin{array}{c}\text { Specific } \\
\text { heat } \boldsymbol{C} \\
(\mathrm{J} / \mathrm{kg} \mathrm{K})\end{array}$ & $\begin{array}{c}\text { Thermal } \\
\text { conductivity } \\
k(\mathrm{~W} / \mathrm{m})\end{array}$ & $\begin{array}{c}\omega \mathrm{b} \\
\left(\mathrm{s}^{-1}\right) * 10^{-3}\end{array}$ & $\begin{array}{c}\mathrm{Qm} \\
(\mathrm{W} / \mathrm{m} 3)\end{array}$ \\
\hline Gland & 1020 & 3060 & 0.322 & $0.34-1.7$ & 700 \\
\hline Tumor & 1020 & 3060 & 0.564 & $6-16$ & 7792 \\
\hline Blood & 1060 & 3840 & & & \\
\hline \hline
\end{tabular}

flow and heat production manifests itself as a ten-fold increase in the perfusion term together with a similar increase in heat production which characterizes the thermal behavior of the cancerous tumor. In vivo measurements were done by Gautherie $^{3}$, who observed a $1.5^{\circ} \mathrm{C}$ temperature difference between the tissue and the average temperature of the blood or, similarly, the temperature difference between the incoming blood and the outgoing blood. Using these data, I obtain for almost all published data ${ }^{4,5,6} Q b>Q m$, which indicates an inconsistency in the data. Similar results are obtained using other data sources. Even though the results are inconsistent, they indicate that the heat generated by diseased tissue is high and most of it is conducted away by the blood flow. As a result, the heat signature will appear away from the cancerous tissue where the blood flows near the skin's surface. Attempts to use thermography as a signature of cancer has had limited success. What I see in a thermogram is an analog to a water-cooled engine. The heat signature is of the radiator and not of the cylinders. As the arteries are at the body core temperature, we will only see hot veins carrying heat from the high metabolic activity area. There are no publications that have shown evidence of collocation between the heat signature and the tumor. Use of IR to image veins in order to identify neovascularization has some utility. Some thermal imaging techniques use what is called "thermal challenge," "coldstress," or "dynamic imaging," where either the breast or some other part of the body is cooled while taking the thermogram. Limited success using this technique was demonstrated by $\mathrm{Y}$ Ohashi and I Uchida, ${ }^{7}$ who subtracted cold images from hot 
ones. Later, independent studies failed to see an advantage in cold challenge without subtraction using visual interpretation ${ }^{8}$. Geetika Dua et al. ${ }^{9}$ showed using a simulation that the high perfusion of the tumor can be detected using heat modulation. The author ${ }^{10,11}$ demonstrated use of the equivalent wave field transform (EWFT) applied to dynamic data to detect the high perfusion associated with the tumor. EWFT was developed as a nondestructive tool and is a linear transform which converts thermal diffusive propagation into a wavelike propagation. The author has shown that heat absorbing high perfusion region acts as a high contrast negative total reflector for heat, similar to reflection from a shorted transmission line. In a transmission line there are forward and backward propagating signals. Since the amplitude at the short is zero, the two signals are equal at that point but of opposite sign or the reflected signal is of reversed polarity. Some moderate success in interpreting breast thermograms has been achieved lately using artificial intelligence. Bardia ${ }^{12}$ et al. used principal component analysis (PCA) applied to dynamic data to identify the veins as preprocessing for AI interpretation. The results are quite similar to direct application of AI to the raw IR images. It appears that AI detection is based on observing neovascularization. A major disadvantage of any methods based on neovascularization is its inability to locate the cancer, it only indicates that cancer's existence, which requires additional testing to localize it.

\section{METHOD}

$A$. The goal is to identify cancer by mapping the temperature of the surface of the breast as a response to external temperature change and identify the signature of the cancer. Thermal mapping of the breast's surface can identify internal heat sources indicating the existence of cancer. The problem is that most of the internal heat is carried away by the veins; thus the true location of the heat source is obscured by heat dissipating through the venous system close to the skin.

$B$. When applying temperature change to the breast, the physiological response can be classified by three processes: First is internal heat sources such as metabolic activity, which is barely changed by the applied external heat. Second is the heat propagation described by the bioheat equation. This propagation is affected mostly by the heat capacity and thermal conductivity of the tissue it propagates through. High perfusion such as that of a tumor, which is a heat sink, can be regarded as a polarity reversing reflector. The third response is that of the veins, which dominates the thermal image. The veins react to the applied thermal change by contracting or dilating. Veins have two important roles: one is the familiar one of returning the blood to the heart, the other is to act as a coolant and thermal regulator. Having high heat capacity and flowing close to the tissue, blood is an ideal coolant. To dispose of the heat, the veins are routed close to the skin. By increasing the vessels' diameter when hot (vasodilation) and decreasing the diameter when cold (vasoconstriction) the body regulates the blood temperature and maintains a constant body core temperature. The normal response to external temperature changes is vasomodulation delayed by propagation time through the tissue.

C. In this analysis I used a sequence of thermal images of the patient's breast following a local temperature change created using a fan. Thermal data were recorded and then analyzed using PCA and independent component analysis (ICA). PCA and ICA are somewhat the opposite of each other. PCA is a technique which reduces the data into a sequence of orthogonal sets while maximizing the variance of each set. It is mainly used to reduce the dimensionality of the data set. ICA is use to separate the data which are comprised of mixed individual channels back into the individual sets by minimization of mutual information and maximization of non-Gaussianity. We can understand the role of PCA and ICA using the following example: We record a video of a blinking face. The first PCA image is of the face with a superposition of the eyes, the following components are different superpositions of the eyes. If we reanalyze those images using ICA, we will recover the original individual eye position. In that case ICA undoes the mixing. Both PCA and ICA have been used in analysis of transient thermal imaging ${ }^{14}$ in nondestructive evaluation (NDE). In NDE a thermal transient reflection, quite similar to the situation in our experiment, is recorded and analyzed. In that case, both PCA and ICA result in very similar images of the defects. It is common to perform PCA prior to ICA as usually the high-order PCA components are mostly noise and can be ignored

\section{EXPERIMENTAL DATA}

Data from the Brazilian Database for Breast Research ${ }^{13}$ with infrared images were obtained by publicly accessible methods. The protocol used for collecting the data: The room temperature was maintained between $20^{\circ} \mathrm{C}$ and $22^{\circ} \mathrm{C}$. A fan was used to cool the skin surface of the breast until it reached $32.5^{\circ} \mathrm{C}$, but for no longer than 5 minutes. The data were sampled every 15 seconds for 5 minutes, for a total of 20 frames. The IR images are captured by a FLIR thermal camera, model SC620, which has a sensitivity of less than $0.04^{\circ} \mathrm{C}$ with a resolution of $640 \times 480$ pixels. These data are data of opportunity i.e., the analysis technique wasn't in mind when the data were collected. Dynamic images were collected from a frontal view while the tumor might have been only visible from a lateral view. A bigger limitation was a lack of medical information -- patients were only classified as "sick" or "healthy" with almost no additional support information. A large number of the patients had no mammography or biopsy while they were still declared sick. The free clinic population is not representative of what we expect to see in a screening population in a developed country. In order to compensate for patient motion, the MATLAB imregister function with a similarity transform followed by an affine transform was applied. It was followed by MATLAB imregdemons. Imregdemons is a transformation which allows for local distortion. The 'AccumulatedFieldSmoothing' parameter was set between 3 and 9 , which is outside the range of values recommended by the package. I register between adjacent images as they are quite similar. I iterate the process a 
few times. One of the reasons for using patient \#273 is the distinct heat pattern which facilitated better registration.

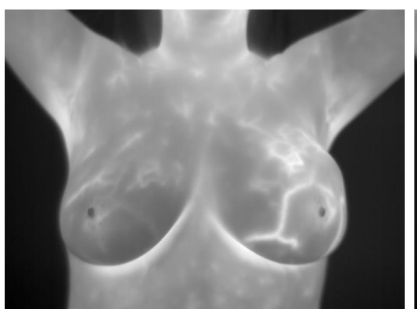

(a)

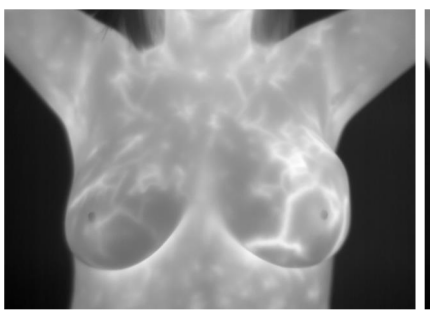

(c)

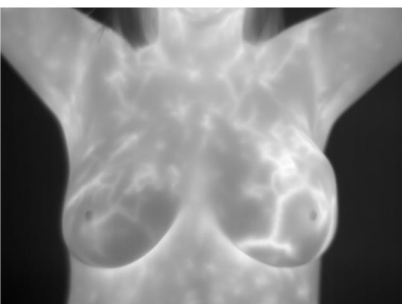

(b)

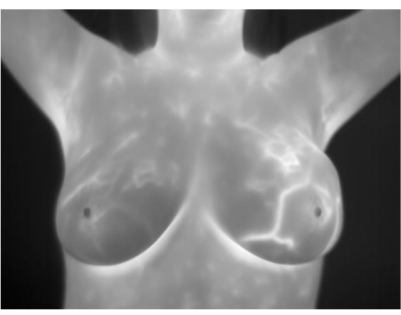

(d)
Figure 1: Thermal images (brighter are hotter) of patient \#274. recorded at (a) $0 \mathrm{sec}$, (b) $75 \mathrm{sec}$, (c) $150 \mathrm{sec}$ and (d) 225sec. Visual inspection reveals no discernable differences between successive images.

\section{ANALYSIS}

I separated the images into left and right breasts and subtract the mean temperature of each set. As all the analysis steps are performed based on individual pixels, I converted the 2D image into a $1 \mathrm{D}$ vector while keeping tabs on the correspondence between the pixels' coordinates and the vector indices. The individual image vectors are stacked together to produce a matrix. I included the static image as the last frame. I kept two sets of data: a masked version of the images where only pixels from regions of interest are included, and the full image. Segmentation was done manually. The transform was calculated using the masked data and applied to the unmasked data. First, we examined patient \#274's left breast. The patient is categorized as "sick" with biopsy on the same breast. The principal component image (e) of the veins dominates the original images. This component has the highest temperature range and the largest number of hot pixels compared to the other images; it is very similar to the individual frames of figure 1 . The contributions of each individual component ((a) to (e)) to the original frame are plotted in figure 3. Inspecting figure 3, we distinguish between smooth monotonic unipolar temperature progression (excluding the last static frame) of components (a), (c), and (e), which also have a higher temperature range. The second group -- images (b) and (d) -has alternating slopes and polarities with a lower temperature range. I believe the first group are images of the heat source, and the second group are images of thermal reflection by high perfusion. Figure 4 presents similar component images of patient \#282. Patient \#282 was categorized as "sick" with

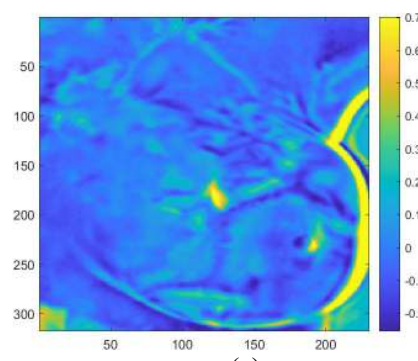

(a)

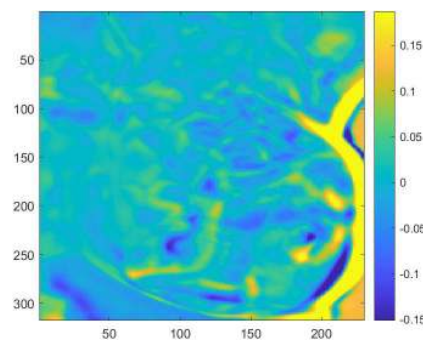

(c)

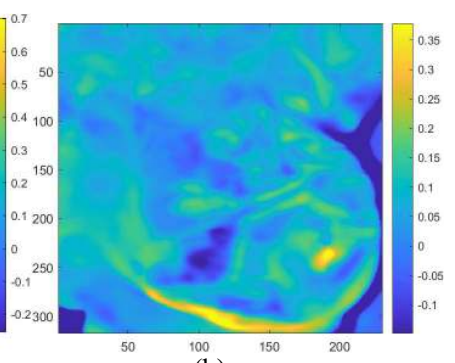

(b)

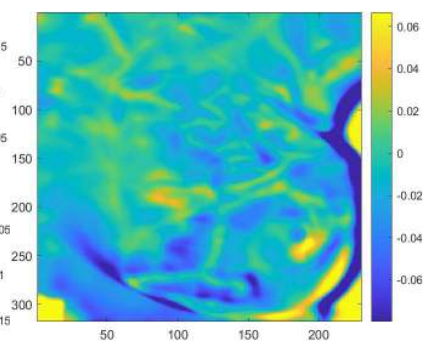

(d)

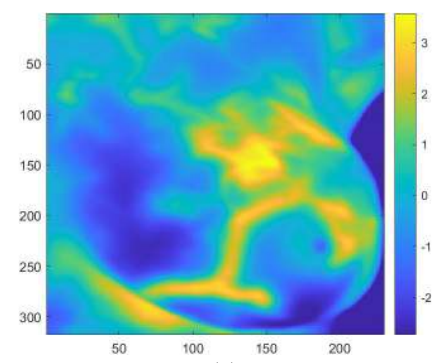

(e)

Figure 2: Component analysis of patient \#274. (a) to (d) are independent components images, (e) is the first PCA image. Temperature in Celsius on the right is normalize to frame 20. The highest temperature is on (e) which we attribute to the veins, next is (a) which we attribute to the metabolic activity.

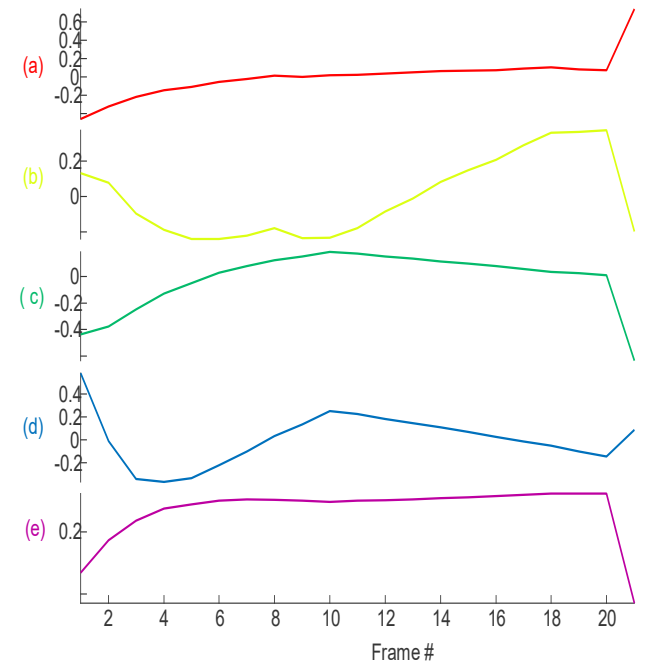

Figure 3: The amplitude contribution of each component (a) to (e) of patient \#274 to original data frame. Last data point is of the static frame. Data is taken at $15 \mathrm{~s} /$ frame. Trace (a) shows minimal temperature dependence, we attribute it to metabolic activity. We attribute traces (b) and (d) to thermal reflection from high perfusion. (e) is the veins response. 


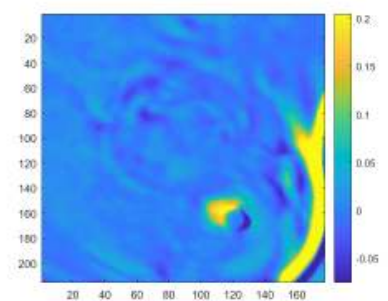

(a)

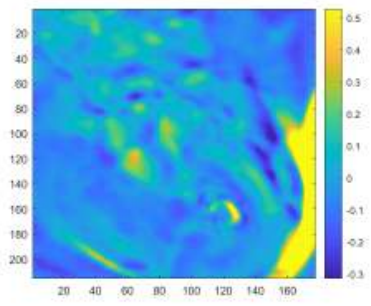

(c)

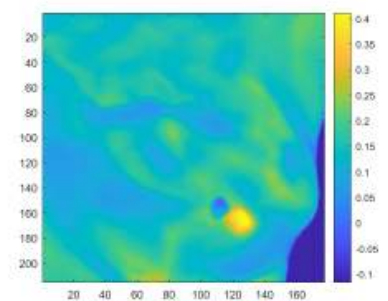

(b)

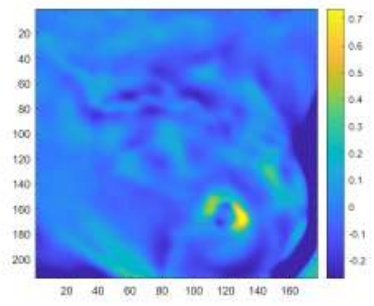

(d

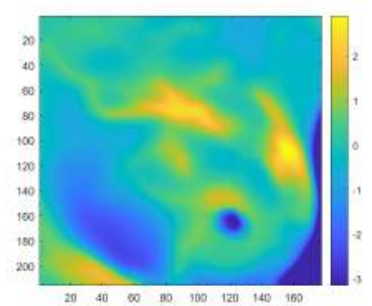

(e) )

Figure 4: Component analysis of patient \#282. (a) to (d) are independent components images, (e) is the first PCA image. Temperature in Celsius on the right is normalize to frame 20. The highest temperature is on (e) which we attribute to the veins, next is (b) which we attribute to the metabolic activity.

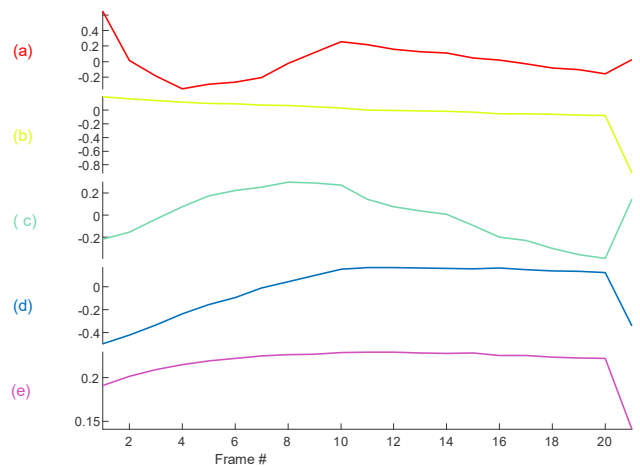

Figure 5: The amplitude contribution of each component (a) to (e) of patient \#282 to original data frame. Last data point is of the static frame. Data is taken at $15 \mathrm{~s} /$ frame, Trace (b) shows minimal temperature dependence, we attribute it to

metabolic activity. We attribute traces (a) and (c) to thermal reflection from high perfusion. (e) is the veins response.

additional information that the tumor is on the left breast on the lower inner quadrant (QIM). Independent component images are labeled (a) to (d). The order of the independent components is inconsequential. The first principal image is shown as (e). The contribution of each component to the original frames is shown in figure 5 plotted in the same order as the components are presented. The temperature scale is to the right of the images.

\section{DISCUSSION}

$A$. This work was motivated by the possibility of distinguishing between direct tumor heat and secondary venous heat using vasomodulation. In term of image clarity, it worked very well. It is hard to draw definite conclusions as the medical information was insufficient. The following conclusions are somewhat speculative. After identifying the veins and removing their contribution from the thermogram, I actually were able to identify another mode: thermal reflection due to tumor high perfusion. Column (e) of plots 3 and 5 are the amplitude of the veins' temperature. We see clearly a similar temperature dependence. Both plots indicate that recording started after the rewarming period was underway. The vasodilation process manifests a time delay; it is composed of a heat propagation delay and a physiological delay followed by similar thermal back propagation delay. We can estimate the thermal delay. For a planar heat front, such as that of the skin surface, the 1D thermal propagation for delta function excitation is given by ${ }^{15}$

$$
\Delta T(t)=\frac{Q}{\sqrt{4 \alpha \pi t}} e^{-\frac{x^{2}}{\alpha t}} \quad \alpha=\mathrm{k} / \rho c
$$

The time to reach its peak is $t_{m}=x^{2} / 2 \alpha t$. If we assume a depth of $1 \mathrm{~mm}$ for the veins in figure 2 or twice that for $x$, using parameters taken from Table 1 for normal tissue, the delay is $25 \mathrm{sec}$, consistent with measurements with only a minimal delay due to physiology. Figure 5 indicates deeper veins on the basis of the time delay confirmed by the diffused shape of image 4 (e). Traces (b) and (d) of figure 3 and (a) and (c) of figure 5, which we interpret as thermal reflection by high prefusion, manifest a time delay of roughly $120 \mathrm{sec}$ which corresponds to about a $5 \mathrm{~mm}$ depth. This depth is consistent with the appearance of a hot spot with clear definition. It is possible to obtain more accurate depth information from the shape of the curve, but that requires either knowledge of the shape of the cooling curve or complete recording of the thermogram from the initiation of the cooling, which we do not have. Total recording of 3 minutes of cooling plus 3 more minutes of decay recording would allow us to resolve the depth up to $1 \mathrm{~cm}$. We can still see deeper by using an image of the metabolic activity: (a) of figure 2 or (d) of figure 4 . Both images have similar companion images (c) and (b) which show a similar unexplained temperature dependence, to understand which would require additional study. Cooling of about one minute with an additional similar time of post recording is sufficient to identify the veins and isolate the tumor heat, but a much longer time is necessary to resolve the reflected heat of the tumor. The model implicitly assumed that the shape of the veins is unchanged, the only change being an increased temperature, as a result of which, the images of patient \#274. figure 2, show a ripple repetition outside the veins. For the shallow heat source in the above cases the image is quite clear. For deeper sources we might need further processing. 


\section{CONCLUSION}

Using data of opportunity, I have shown that employing principal and independent components analysis we can decompose sequences of dynamic breast images into components which appear to be the tumor direct heat, secondary vein heat which dominates the original image, and reflected heat due to high perfusion. We have to remember that these data were optimized for AI interpretation. This dataset contains multiple static views but only one single dynamic view. It is possible that the tumor will be invisible in that single view. For the AI analysis, sick or healthy characterization was sufficient, but our method, which localizes the tumor, requires more information. While the direct heat from the tumor was always regarded as the objective of breast thermography, thermography actually shows us images of the veins close to the skin. We have seen lately a flurry of breast thermal modeling with improved geometrical details, but all of them ignore the elephant in the room -- the heat from the veins. Unless we can remove the heat from the veins, those modeling efforts are just an academic exercise. In this work we showed that based on vasomodulation one can differentiate between the desired heat of the tumor and the secondary heat from the veins. I also identified the reflected heat resulting from the high perfusion of the tumor.

\section{ACKNOWLEDGMENT}

I would like to thank Dr. Mark Mandelkern and Dr. Paul F. Schippnick for critical reading and suggestions. And Dr. Jonathan P. Gershenson for useful discussions.

\section{REFERENCES}

[1] Wissler EH. Pennes' 1948 paper revisited. Journal of Applied Physiology. 1998;85:35-41. doi: 10.1152/jappl.1998.85.1.35.

[2] Azarnoosh J, Hassanipour F. Determining the thermal characteristics of breast cancer based on high-resolution infrared imaging, 3D breast scans, and magnetic resonance imaging. Sci Rep. 2020 Jun 22;10(1):10105. doi: 10.1038/s41598-020-66926-6. PMID: 32572125; PMCID:

[3] Gautherie M. Thermopathology of breast cancer: measurement and analysis of in vivo temperature and blood flow. Ann N Y Acad Sci. 1980;335:383-415. doi: 10.1111/j.1749-6632.1980.tb50764.x. PMID: 6931533.

[4] Gonzalez-Hernandez J-L, Kandlikar SG, Dabydeen D, Medeiros L, Phatak P. Generation and thermal simulation of a digital model of the female breast in prone position. Journal of Engineering and Science in Medical Diagnostics and Therapy. 2018;1:041006. doi: 10.1115/1.4041421E.

[5] Ng EYK, Sudharsan NM. Computer simulation in conjunction with medical thermography as an adjunct tool for early detection of breast cancer. BMC Cancer. 2004;4:17. doi: 10.1186/1471-2407-4-17
[6] Azarnoosh J, Hassanipour F. Determining the thermal characteristics of breast cancer based on high-resolution infrared imaging, 3D breast scans, and magnetic resonance imaging. Sci Rep. 2020 Jun 22;10(1):10105. doi: 10.1038/s41598-020-66926-6. PMID: 32572125; PMCID: PMC7308290

[7] Ohashi Y, Uchida I. Applying dynamic thermography in the diagnosis of breast cancer. IEEE Eng Med Biol Mag. 2000 May-Jun;19(3):42-51. doi: 10.1109/51.844379. PMID: 10834115.

[8] Amalu WC. Nondestructive testing of the human breast: the validity of dynamic stress testing in medical infrared breast imaging. Conf Proc IEEE Eng Med Biol Soc. 2004;2004:1174-7. doi: 10.1109/IEMBS.2004.1403375. PMID: 17271894.

[9] Dua G, Mulaveesala R. Applicability of active infrared thermography for screening of human breast: a numerical study. J Biomed Opt. 2018 Mar;23(3):1-9. doi: 10.1117/1.JBO.23.3.037001. PMID: 29560626.

[10] J. Gershenson and M. Gershenson "Use of equivalent wave field transform in evaluating dynamic thermal tomography of infrared breast

images", Proc. SPIE 11004, Thermosense: Thermal Infrared Applications XLI, 1100404 (8 May 2019); https://doi.org/10.1117/12.2517415

[11]J. Gershenson and M. Gershenson "Early results for equivalent wavefield transform as a direct solution to the inverse modeling problem for active infrared thermography and potential for perfusion information to differentiate healthy versus cancerous breast tissue" Proc. SPIE 11312, Medical Imaging 2020: Physics of Medical Imaging, 113125E (16 March 2020); doi: $10.1117 / 12.2548218$

[12] Bardia Yousefi, OrcID,Hamed Akbari and Xavier P.V. Maldague, "Detecting Vasodilation as Potential Diagnostic Biomarker in Breast Cancer Using Deep Learning-Driven Thermomics" Journals Biosensors Volume 10 Issue 11 10.3390/bios10110164 (31 October 2020).

[13] L. F. Silva; D. C. M. Saade; G. O. Sequeiros; A. C. Silva; A. C. Paiva; R. S. Bravo and A. Conci, "A New Database for Breast Research with Infrared Image, Journal of Medical Imaging and Health Informatics”, 4 (1), 2014. http://visual.ic.uff.br/en/proeng/

[14] Yi Liu1, Jin-Yi Wu2, Kaixin Liu1, Hsiu-Li Wen2, Yuan Yao5,2, Stefano Sfarra5,3 and Chunhui Zhao4. "Independent component thermography for nondestructive testing of defects in polymer composites", Measurement Science and Technology, Volume 30, Number 4 
[15] Carslaw, H. S., and J. C. Jaeger (1959), Conduction of Heat in Solids, 2nd Edition, Clarendon, Oxford. 